\title{
Gold nanoparticle-based miR I 55 antagonist macrophage delivery restores the cardiac function in ovariectomized diabetic mouse model
}

This article was published in the following Dove Press journal:

International Journal of Nanomedicine

II July 2017

Number of times this article has been viewed

Chengming Jia, ${ }^{1,2, *}$ Hui

Chen, ${ }^{3, *}$ Mengying Wei, ${ }^{2, *}$

Xiangjie Chen, ${ }^{4}$ Yajun

Zhang, ${ }^{5}$ Liang Cao,' Ping

Yuan,' Fangyuan Wang,' Guodong Yang, ${ }^{2}$ Jing $\mathrm{Ma}^{\prime}$

'Department of Chinese Medicine, Xijing Hospital, The Fourth Military Medical University, Xi'an, China;

${ }^{2}$ Department of Biochemistry and Molecular Biology, The Fourth Military Medical University, Xi'an, China; ${ }^{3}$ Department of Plastic Surgery, Tangdu Hospital, The Fourth Military Medical University, Xi'an, China; ${ }^{4}$ Department of Mathematics, Southeast University, Nanjing, China; ${ }^{5}$ Department of Ultrasound Diagnosis, Tangdu Hospital, The Fourth Military Medical University, Xi'an, China

*These authors contributed equally to this work
Correspondence: Jing Ma

Department of Chinese Medicine,

Xijing Hospital, The Fourth Military

Medical University, 127 Changlexi Road,

Xi'an 710032, China

Tel +8602984775349

Email jingma@fmmu.edu.cn

Guodong Yang

Department of Biochemistry and Molecular

Biology, The Fourth Military Medical University,

169 Changlexi Road, Xi'an, 710032, China

Tel +8602984774516

Email yanggd@fmmu.edu.cn
Abstract: Diabetic cardiomyopathy is a common disease in postmenopausal women, in whom the estrogen deficiency aggravates the pathology. In this study, we have found that estrogen deficiency due to ovariectomy aggravates the inflammation in the hearts of diabetic mice, as depicted by excessive proinflammatory type 1 macrophages (M1) over anti-inflammatory type 2 macrophages (M2). Accordingly, an additional increase of reactive oxygen species, cell apoptosis, cardiac hypertrophy, and fibrosis was observed in the hearts of ovariectomized diabetic mice, in comparison with the diabetes-only group. Significantly, miR155, a potent promoter of M1 polarization, was found to be additionally enhanced in the macrophages and hearts by ovariectomy. Tail vein injection of miR155-AuNP, in which thiol-modified antago-miR155 was covalently conjugated with gold nanoparticle (AuNP), preferentially delivered the nucleic acids into the macrophages via phagocytosis. Together with the increased M2 ratio and reduced inflammation, in vivo delivery of antago-miR155 reduced cell apoptosis and restored the cardiac function. The restoration efficacy of miR155-AuNP was much better than general macrophage depletion by clodrosome. In summary, we revealed that M1/M2 imbalance contributes to the aggravated cardiomyopathy in ovariectomized diabetic mice, and therapeutically reducing miR155 in macrophages by AuNP serves as a promising strategy in improving cardiac function.

Keywords: gold nanoparticle, miR155, cardiac function, macrophage, ovariectomized diabetic mouse model, targeted delivery

\section{Introduction}

Cardiovascular disease, especially cardiomyopathy, is the leading cause of morbidity and mortality all over the world. ${ }^{1}$ It is well known that diabetes is an independent risk factor for cardiovascular disease. ${ }^{2}$ Notably, the prevalence of diabetic cardiovascular disease significantly increases in postmenopausal women. Although estrogen deficiency is found to aggravate cardiac defects in postmenopausal women, ${ }^{3,4}$ the detailed underlying mechanism remains not fully understood.

Inflammation is one of the accompanying key traits of cardiomyopathy. Macrophages are the key immune cells regulating inflammation in the context of cardiovascular diseases. ${ }^{5,6}$ Upon various environmental cues (eg, microbial products, damaged cells, and activated lymphocytes) or under different pathophysiologic conditions, macrophages undergo a different phenotypic polarization. ${ }^{7}$ There are at least two types of macrophages, the proinflammatory type 1 macrophage (M1 type macrophage) and the anti-inflammatory type 2 macrophage (M2 type macrophage). M1 type macrophages mainly produce proinflammatory factors, and excessive M1 macrophages would cause inflammation and thus tissue damage. In contrast, 
M2 type macrophages mainly produce anti-inflammatory factors, inhibiting inflammation and promoting tissue repair. ${ }^{8}$ Although multiple studies have found that the balance between M1 and M2 plays an important role in cardiovascular diseases, ${ }^{9,10}$ the detailed role of M1/M2 in postmenopausal diabetic patients remains unknown.

miRNAs, which are a class of 20-24 nt-long small noncoding RNAs, posttranscriptionally regulate the gene expression via base pairing interaction with the $3^{\prime} \mathrm{UTR}$ of the target mRNAs. ${ }^{11}$ Recently, miRNAs were found to be able to regulate macrophage phenotype commitment in the course of various diseases. For example, miR155 can directly block IL-13-induced macrophage M2 phenotype via suppressing the expression of IL-13R $\alpha 1 .{ }^{12}$ Corsten et al recently established that miR 155 was upregulated in the heart during viral infection, contributing to the adverse inflammatory response. ${ }^{13}$ Meanwhile, the pharmacological inhibition of miR-155-5p utilizing anti-miRs (chemically modified antisense oligonucleotides) reduced the cardiac infiltration of monocyte/macrophages, attenuated myocardial damage, and improved cardiac function and mortality. ${ }^{13}$ All of these results suggest that the therapeutic targeting of miR155 may benefit cardiovascular disease patients by dampening inflammation. However, the question whether miR155 is involved in the postmenopausal diabetic cardiovascular disease and how to precisely deliver miR155 modulators to the macrophages remain unknown.

Gold nanoparticles (AuNPs) are nanoscale gold particles, often in the form of colloid with a diameter between 1 and $200 \mathrm{~nm}$. AuNPs serve as attractive materials for nucleic acid delivery, ${ }^{14,15}$ mainly because of the following advantages. First, AuNPs can be fabricated in a scalable fashion with low-size dispersity. ${ }^{16}$ Second, multifunctional monolayers can be easily achieved on the AuNP surface, allowing multiple functional moieties, such as nucleic acids and targeting agents, to be conjugated on the particle surface. ${ }^{17,18}$ In addition, the cytotoxicity, ${ }^{19,20}$ biodistribution, ${ }^{21}$ and in vivo excretion properties ${ }^{22}$ can be modulated by tuning the particle size and surface functionality.

In this study, we have explored the role of macrophage polarization and the potent regulator miR155 in cardiac dysfunction in an ovariectomized (OVX) diabetic mouse model. We have shown that miR155 upregulation contributes to excessive inflammation and thus cardiac deficiency via promoting M1 polarization. AuNP conjugated with miR155 antagonist can ideally and preferentially deliver the nucleic acids into macrophages via phagocytosis, which in turn restores the cardiac function. Our study implies that AuNPs conjugated with antago-miR155 might represent an ideal strategy to improve the cardiovascular disease prognosis in postmenopausal diabetic patients.

\section{Materials and methods Animals}

All the mice were bred in the Fourth Military Medical University Research Animal Facility and all procedures involving animal handling were in accordance with the guidelines of the Fourth Military Medical University, which adheres to Guide for the Care and Use of Laboratory Animals. All the experimental protocols were approved by the Institutional Animal Care and Use Committee of the Fourth Military Medical University. C57BL/6 mice were housed in plastic cages in a controlled environment animal facility at $22^{\circ} \mathrm{C} \pm 2^{\circ} \mathrm{C}$ with a 12 -hour light/dark cycle. They were acclimatized for 1 week and given a standard mouse chow pellet with ad libitum water access.

The mice were OVX, as previously described. ${ }^{39}$ Briefly, 8-week-old female mice were anesthetized using $1 \%$ sodium pentobarbital solution $(30 \mathrm{mg} / \mathrm{kg}$, injected intraperitoneally). A $1-\mathrm{cm}$ incision was made in the skin and back muscles parallel to the midline of the animal. Next, the ovaries were located, and the oviduct, including the ovarian blood vessels, was ligated, followed by ovary removal. The incision in the back musculature was closed with a nylon thread (Ethicon 4.0) and the skin was sutured with stainless steel wound clips. Throughout the surgical procedure, mouse body temperature was maintained at $37^{\circ} \mathrm{C}$ with a heating pad. One surgeon performed all the procedures.

Streptozotocin (STZ) (Sigma, Darmstadt, Germany) was used to produce experimental diabetes. One week after undergoing OVX, C57/BL6 mice were injected intraperitoneally with a low dose of STZ $(50 \mathrm{mg} / \mathrm{day} / \mathrm{kg}$ body weight, dissolved in fresh cold citrate buffer [pH 4.5]) for 5 consecutive days. Hyperglycemia (glucose $>22 \mathrm{mmol} / \mathrm{L}$ ) was confirmed 2 weeks later using a reflectance meter. For tissue harvest, mice were anesthetized with isoflurane and sacrificed by cervical dislocation. Hearts, livers, and spleens were quickly removed and frozen in an optimal cutting temperature (OCT) compound.

\section{Cell culture and transfection}

RAW264.7 cells were purchased from the American Type Culture Collection (ATCC TIB-71, Manassas, VA, USA) and maintained in RPMI 1640 supplemented with 10\% heatinactivated FBS, $2 \mathrm{mM}$ glutamine and $100 \mathrm{U} / \mathrm{mL}$ penicillin, and $100 \mu \mathrm{g} / \mathrm{mL}$ streptomycin (Gibco, Carlsbad, CA, USA). Cell cultures were maintained at $37^{\circ} \mathrm{C}, 5 \% \mathrm{CO}_{2}$ atmosphere. About $6 \mu \mathrm{L}$ lipofectamine 2000 (Invitrogen, Carlsbad, CA, 
USA) was mixed with $10 \mu \mathrm{L}$ miR155 mimics/inhibitor or negative control at room temperature for about half an hour. Then, RAW264.7 cells were infected with the mixture of the above for 5 hours according to the manufacturer's protocol. The media were discarded, and the cells were washed twice and replaced with fresh RPMI 1640 supplemented with 10\% FBS. After additional 72-hour culture plus-indicated treatments, the total RNA from the transfected cells was isolated for a quantitative polymerase chain reaction (qPCR) analysis of mRNA/miRNA, as described below.

\section{TUNEL assay}

Apoptotic nuclei were probed using a terminal deoxynucleotidyl transferase mediated dUTP nick end labeling (TUNEL) assay, as previously reported..$^{40}$ The frozen section was incubated in 4\% paraformaldehyde (PFA) for $20 \mathrm{~min}$. This section was then washed in PBS two times and dried. Reaction solution (TUNEL kits; Promega, Madison, WI, USA) was added to each section and the reactions were performed at $37^{\circ} \mathrm{C}$ for $60 \mathrm{~min}$ in the dark. After incubation, samples were again washed three times in PBS. Hoechst 33258 (Hoechst) was used to stain the nuclei. Apoptotic index, expressed as a percentage of TUNEL-positive nuclei to total nuclei, was calculated for each section.

\section{Histology and immunohistochemistry}

To measure the cross-sectional area of individual cardiac myocytes in frozen short-axis sections of mouse hearts, Oregon Green 488-conjugated wheat germ agglutinin (WGA) was used as in routine immunohistochemical analysis. ${ }^{41}$ The incubation time was 1 hour protected from light. After three washing steps, the sections were labeled by Hoechst diluted 1:1,000 in PBS. Vascular endothelial cells and macrophages were stained in OCT-embedded hearts by immunofluorescence staining using rat antiCD31 primary antibody (BD Pharmingen, San Jose, CA, USA) and antimouse CD68 primary antibody (Abcam, Cambridge, UK), respectively, followed by $\mathrm{Cy} 3$-conjugated goat antirat and goat antimouse secondary antibody (Life Technologies, Carlsbad, CA, USA) as before. ${ }^{42}$ Briefly, 10 - $\mu \mathrm{m}$-thick OCT-embedded cryosections were fixed with $4 \%$ PFA, permeabilized with $0.2 \%$ Triton X100, and blocked with $4 \%$ bovine serum albumin. Sections were then incubated with primary antibody overnight $\left(4^{\circ} \mathrm{C}\right.$ in a humidified chamber) and then washed and incubated with secondary antibody for 1 hour at $37^{\circ} \mathrm{C}$. After these processes, the sections were visualized and images captured using a fluorescence microscope, and CD31-positive vessels were quantified (ImageJ) and reported as percent per field. CD68-positive area was also quantified (ImageJ) and reported as "percent per field".

\section{Echocardiography}

A C57/BL6 mouse was anesthetized using 2.5\% isoflurane in an anesthesia induction box and maintained in 1.0\%-1.5\% isoflurane during the echocardiography study. The mouse was laid on the operating table, with left thorax wiped clean with depilatory cream. The mouse was then fixed in the supine position and smeared with an ultrasonic coupling agent before echocardiography examination. The left thorax was selected to view the left ventricular long-axis and shortaxis section for ultrasonic testing. Three consecutive cardiac cycles were recorded. End-diastolic left ventricular and right ventricular diameter, left ventricular end-systolic volume, and end-diastolic volume were recorded and left ventricular ejection fraction (LVEF) and left ventricular fraction shortening (LVFS) were calculated.

\section{Masson staining}

The fresh cardiac tissue was immediately fixed in 10\% formaldehyde. After tissue processing and paraffin embedding, cross-sectional slices were obtained. In the last stage, they were stained with Masson trichrome. Green staining is the area of collagen fibrosis, while red stains cell contents.

\section{Real-time-qPCR analysis of mRNA}

Total RNA from the heart or macrophage cell line RAW264.7 was isolated using TRIzol ${ }^{\circledR}$ reagent (Invitrogen) according to the manufacturer's protocol. cDNA was synthesized according to the instructions of reverse transcription kits (Prime Script RT Reagent Kit, TaKaRa, Dalian, China). The cDNA samples were then used for real-time PCR measurement with target-specific primers. Real-time PCR was performed on the CFX96 system (Bio-Rad, Hercules, CA, USA) using SYBR Green I (TakaRa). The following primers were used in the PCR experiments: IL-1 $\beta$, forward primer: 5'-GCAACTGTTCCTGAACTCAACT-3', reverse primer: 5'-ATCTTTTGGGGTCCGTCAACT-3', IL-10, forward primer: 5'-TTTAGGCGAGAAGCTGAAGG-3', reverse primer: 5'-TCTTCACAGGGCAGGAATCT-3', $\beta$-actin, forward primer: 5'-CTGTATTCCCCTCCATCGTG-3', and reverse primer: 5'-TACTCCTGCTTGCTGATCCA-3'. All the reactions were done at least in triplicate. The reaction was as follows: $95^{\circ} \mathrm{C}$ for $3 \mathrm{~min}$, then with repeated 45 cycles of $95^{\circ} \mathrm{C}$ for $5 \mathrm{~s}, 60^{\circ} \mathrm{C}$ for $30 \mathrm{~s}, 72^{\circ} \mathrm{C}$ for $30 \mathrm{~s}$, and finally, $72^{\circ} \mathrm{C}$ for $30 \mathrm{~s}$. The threshold cycles $(\mathrm{Ct})$ were calculated automatically using the CFX manager software (Bio-Rad). Relative gene expression was calculated by $2^{-\Delta \Delta \mathrm{Ct}}$. 


\section{qPCR analysis of miRNA}

Poly-A real-time-qPCR was performed to validate miRNA expression. ${ }^{43}$ Briefly, $2 \mu \mathrm{g}$ of the total RNA fraction isolated using TRIzol reagent (Invitrogen) from heart or RAW264.7 macrophages was polyadenylated with E. coli Poly(A) Polymerase for $60 \mathrm{~min}$ at $37^{\circ} \mathrm{C}$ in a $20-\mu \mathrm{L}$ reaction volume. Then, cDNA was synthesized from $100 \mathrm{ng}$ of polyadenylated small RNAs in a $20-\mu \mathrm{L}$ reaction volume. Reverse transcription was performed for $60 \mathrm{~min}$ at $37^{\circ} \mathrm{C}$. Real-time quantitative PCR was performed using miRcute Plus miRNA qPCR Detection Kit (SYBR Green) (TIANGEN, Beijing, China). The following primers were used in the PCR experiments: miR155, forward primer: 5'-AATGCTAATTGTGATAGGGGT-3', reverse primer was provided in the kit; U6, forward primer: 5'-CTCGCTTCGGCAGCACA-3', reverse primer: 5'-AACGCTTCACGAATTTGCGT-3'. PCR conditions were same as above, and relative expression was calculated by $2^{-\Delta \Delta C t}$.

\section{Gold nanoparticle-mediated siRNA delivery}

Thiolated control (5'-AUCGAAUUCCUGCAGCCCG UUAAAAGAAAAAGAAAAGAA-thiol-3') or miR155 antagonist (5'-ACCCCUAUCACGAUUAGCAUUAAAA GAAAAAGAAAAGAA-thiol-3') oligonucleotides were dissolved in $\mathrm{H}_{2} \mathrm{O}$ with $0.15 \mathrm{M}$ sodium phosphate buffer.

Twenty-four $\mu \mathrm{L}$ of the above solution was incubated with $1 \mathrm{~mL}$ of standard AuNPs for 1 hour at room temperature. The mixture was aged by increasing the salt concentration (from 0.1 to $0.3 \mathrm{M} \mathrm{NaCl}$ ) and sonicating to increase the coverage of oligonucleotides on the surface of $\mathrm{Au}$, which is achieved by adding $1 \mathrm{M} \mathrm{NaCl}$ stepwise in $10-\mu \mathrm{L}$ increments with 5-min incubation time in between until reaching a final $\mathrm{NaCl}$ concentration of $100 \mathrm{mM}$. After incubating at $4^{\circ} \mathrm{C}$ for 16 hours, siRNA gold conjugates were pelleted by centrifuging at $17,000 \times \mathrm{g}$ for $30 \mathrm{~min}$ and resuspended in $200 \mu \mathrm{L} 10 \mathrm{mM}$ sodium phosphate buffer. The size distribution of the AuNP was analyzed by Nanoparticle Tracking Analysis (Malvern, PA, USA).

\section{Analysis of distribution of AuNP-siRNA in vivo}

For in vivo uptake analysis, Cy3-labeled siRNAs (5'-Cy3-AU CGAAUUCCUGCAGCCCGUUAAAAGAAAAAGAAA AGAA-thiol-3') were conjugated with AuNPs using the same method as above. Then, the AuNPs were injected via tail vein. After 48 hours, the mice were harvested and processed for Hoechst nuclear staining. The $\mathrm{Cy} 3$ and nuclear staining were observed under a fluorescent microscope.

\section{Macrophage depletion with clophosome}

For macrophage depletion, clodronate liposome (clophosome, $10 \mu \mathrm{L} / \mathrm{g}$, twice a week) was injected though the tail vein. To confirm the depletion efficiency, the mice were sacrificed and the tissues were harvested for CD68 (macrophages cell marker) staining and Hoechst for nuclei staining. The images were taken under the fluorescent microscope.

\section{Lysosome staining}

Lyso-Tracker Red, a kind of lysosome red fluorescent probe, was used to probe lysosomes in living cells. ${ }^{44}$ After removing the growth medium, the cells were incubated together with Lyso-Tracker Red working liquid (50 $\mathrm{nM}$, diluted in DMEM) for 2 hours. Then, the cells were washed with PBS, and Hoechst was used to stain the nuclei. The cells were examined using a confocal microscope, and representative pictures were photographed.

\section{Statistical analysis}

All data are expressed as mean \pm SD or mean \pm SEM, unless otherwise stated. The statistical significance was determined by Student's $t$-test or ANOVA. $P<0.05$ was considered to be statistically significant.

\section{Results}

\section{Aggravated cardiac dysfunction in OVX diabetic mice}

To characterize the cardiac dysfunction in postmenopausal diabetic patients, we established an OVX diabetic mouse model and subsequent five sequential injections of STZ within 1 week (Figure 1A). Ten weeks later, cardiac function and histology were systematically analyzed. Echocardiographic analyses showed that the systolic cardiac function, LVEF and LVFS were decreased in the OVX group, diabetic group, and OVX diabetic group, when compared with the normal control group (Figure 1B, E, and F), with the lowest systolic cardiac function in the OVX diabetic group. Meanwhile, the diastolic function was also decreased in the above three groups (data not shown). Consistently, a more obvious cardiac hypertrophy and an aggravated fibrosis were observed in the OVX diabetic group, as seen from the larger cellular size (Figure 1C and G) and larger blue area of Masson staining in the OVX diabetic group (Figure 1D and H). Besides, the vascular density in the OVX diabetic group also decreased significantly (Figure S1A and B). All of these findings suggest that the OVX diabetic mice displayed an aggravated cardiac dysfunction. 


\section{Augmented inflammation accounts for the cardiac dysfunction}

To further unravel the underlying cellular and molecular mechanisms for the aggravated cardiac dysfunction, we next explored the role of apoptosis and inflammation in the whole process. As expected, increased apoptosis was observed in the hearts of OVX diabetic mice (Figure S2A and $\mathrm{B})$. To find the dominant factors coordinating cell

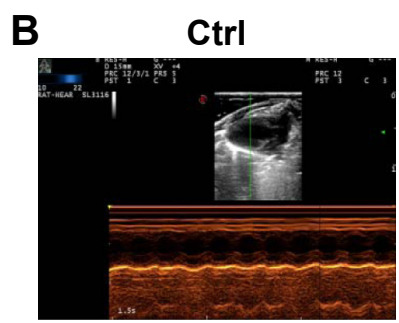

C
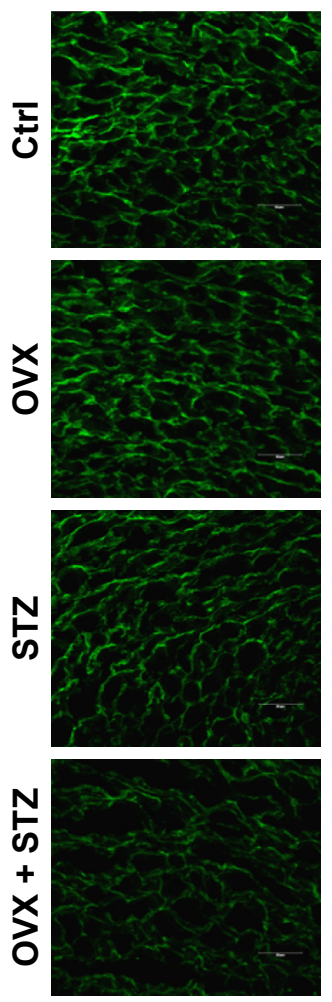

E
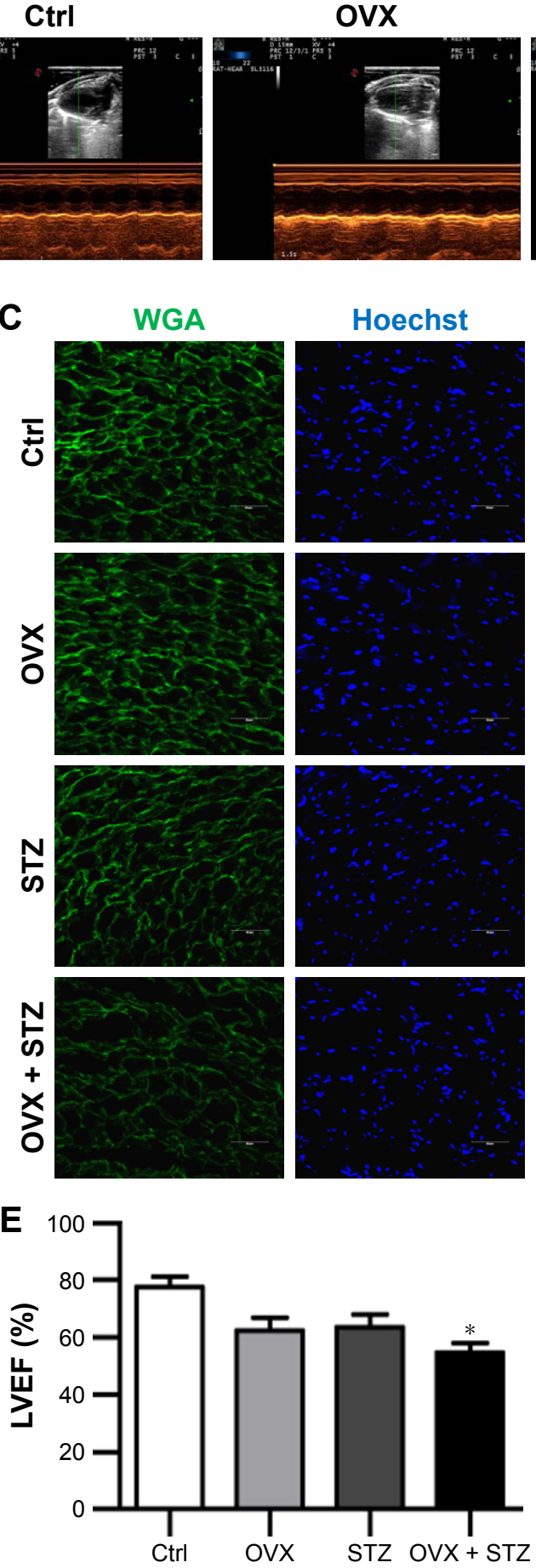

Morphology, morphometry, fluorescence microscopy

10 weeks

STZ

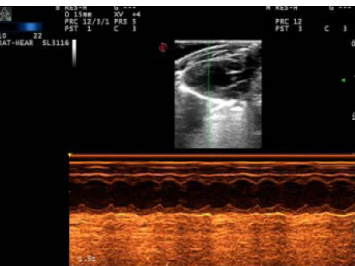

OVX + STZ
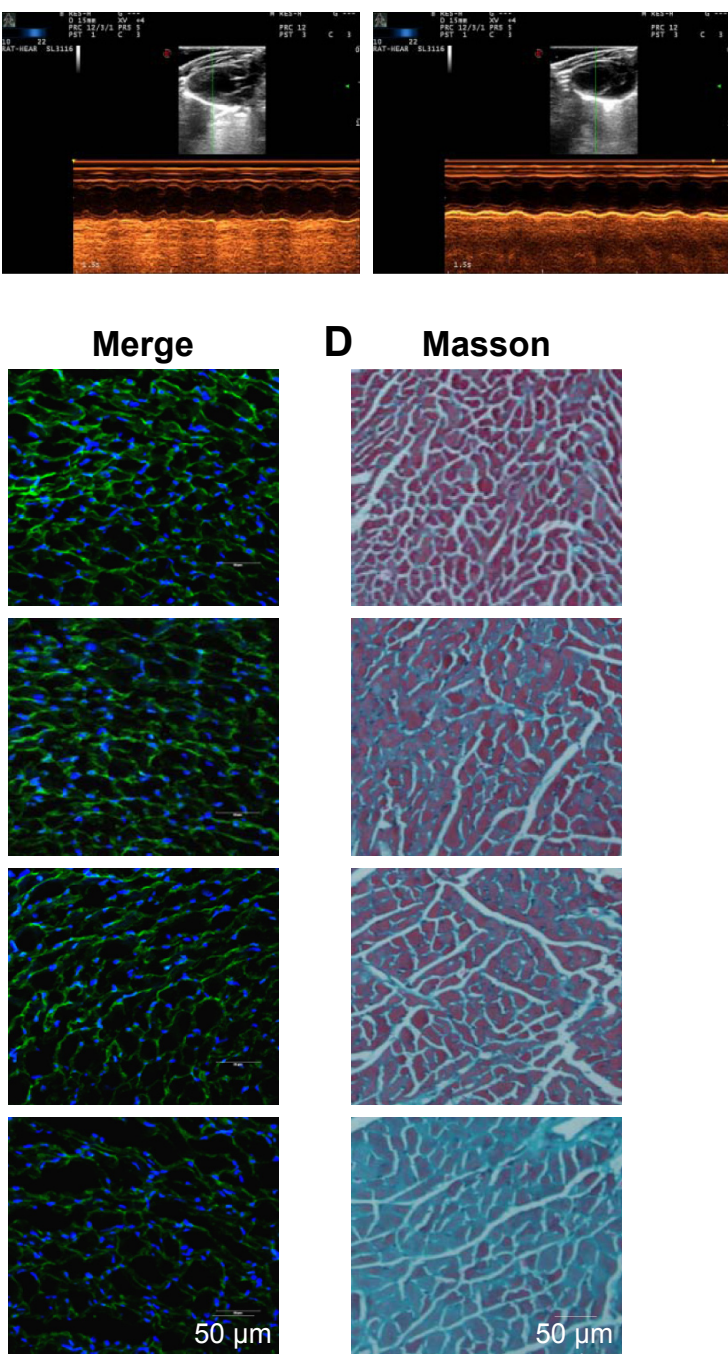

$\mathbf{F}$

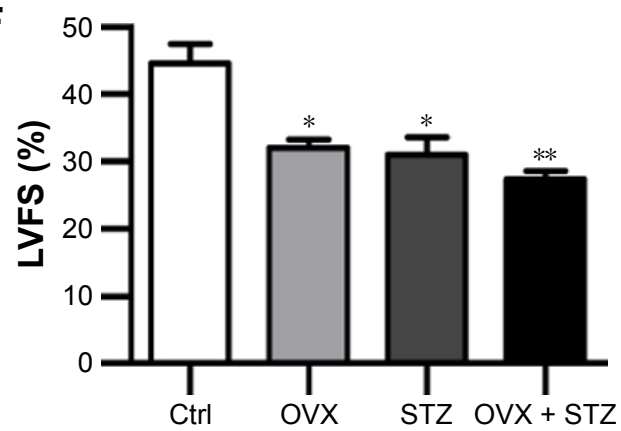

Figure I (Continued) 

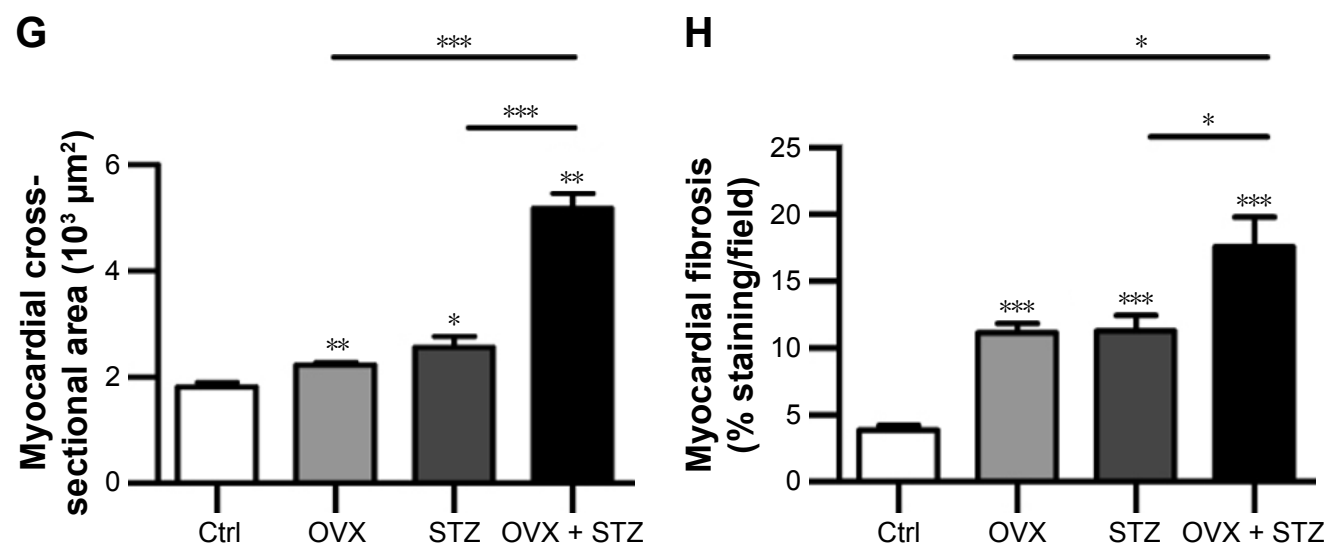

Figure I Ovariectomy aggravates cardiomyopathy in diabetic mice.

Notes: (A) Schematic representation of the experimental procedure. OVX and sham female mice were subjected to STZ or vehicle treatment I week after operation. Ten weeks later, cardiac function, morphology, and cellular changes were systematically analyzed. (B) Representative images of the M-mode echocardiography of control, OVX, diabetic, and OVX diabetic mice before sacrifice. (C) Representative WGA-stained sections from the left ventricular mid-chamber of OVX, diabetic, and OVX diabetic mice. (D) Masson Trichrome staining of the hearts from OVX, diabetic, and OVX diabetic mice. (E) LVEF value of cardiac systolic function in the OVX, diabetic, and OVX diabetic mice groups (mean $\pm S D, n=5$ mice in each group, $* P<0.05$ ). (F) LVFS value of cardiac systolic function in the OVX, diabetic, and OVX diabetic goups (mean $\pm S D, n=5$ mice in each group, $* P<0.05$, $* * P<0.01$ ). (G) Quantitative data of the cardiomyocyte size in the indicated groups (mean $\pm S E M$, analyzed from 4 representative sections from 4 mice in each group, $* P<0.05$, $* * P<0.0 \mathrm{I}$, and $* * * P<0.00 \mathrm{I}$ ). (H) Quantitative data of the fibrotic area in the indicated groups (mean $\pm S E M$, analyzed from 4 representative sections from 4 mice in each group, $* P<0.05$ and $* * * P<0.00 I$ ). Scale bars $=50 \mu \mathrm{m}$ and apply to all images.

Abbreviations: Ctrl, control; LVEF, left ventricular ejection fraction; LVFS, left ventricular fraction shortening; OVX, ovariectomized; SEM, standard error of the mean; STZ, streptozotocin; WGA, wheat germ agglutinin.

apoptosis, angiogenesis, and fibrosis, we focused on the macrophages, especially M1 and M2 polarization. We thus explored the infiltration of macrophages in the hearts of OVX diabetic mice. As expected, there were more CD68+ staining in the hearts of OVX diabetic mice, in comparison with that in the normal control, diabetes, or ovariectomy-only group (Figure 2A and $\mathrm{B}$ ). In addition, a qPCR analysis revealed that the $\mathrm{M} 2$ phenotype marker genes (IL-10) were repressed, whereas M1 marker genes (IL-1 $\beta$ and miR155) were increased in the OVX diabetic mice (Figure 2C-E), suggesting that imbalance between M1 and M2 might play an essential role in the process.
A
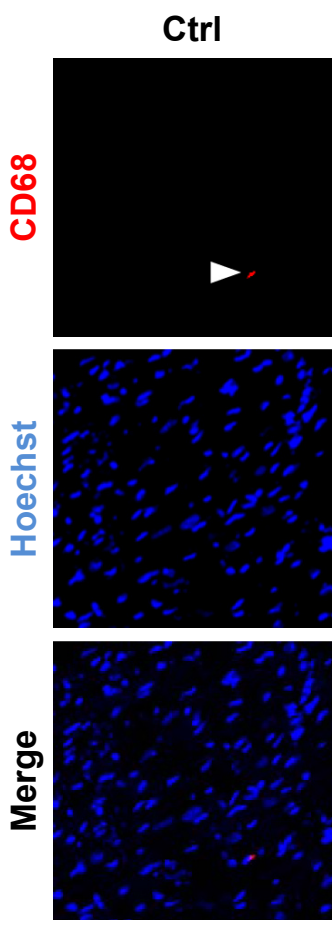

ovX
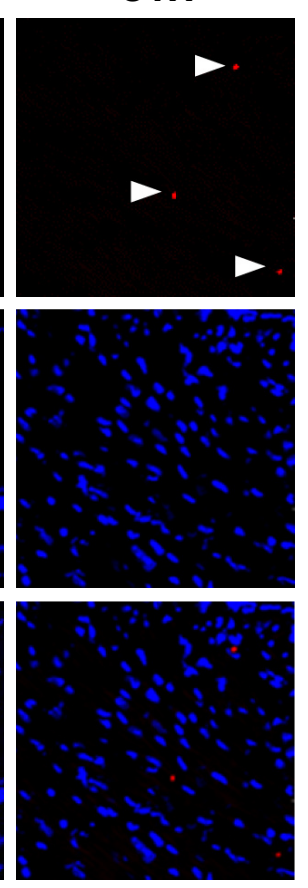

STZ
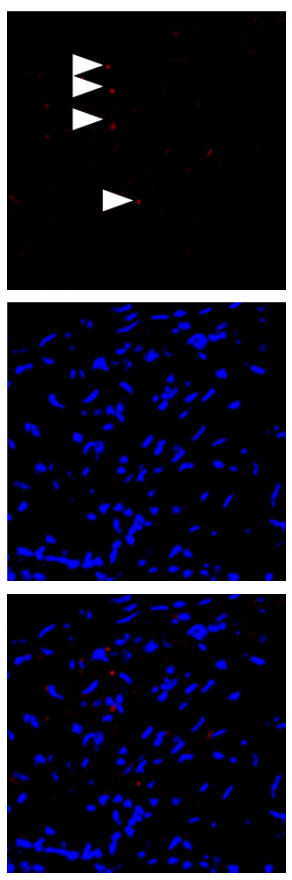

OVX + STZ
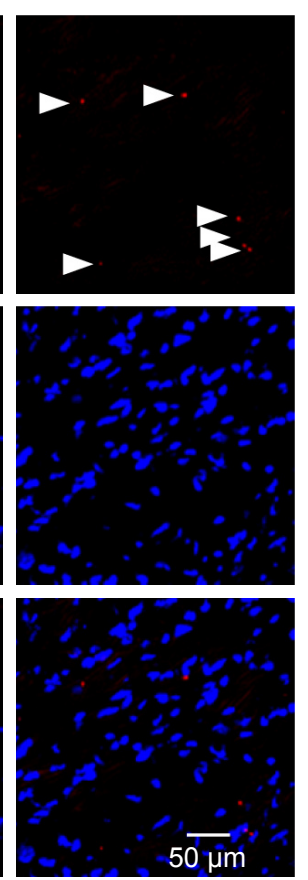

Figure 2 (Continued) 
B
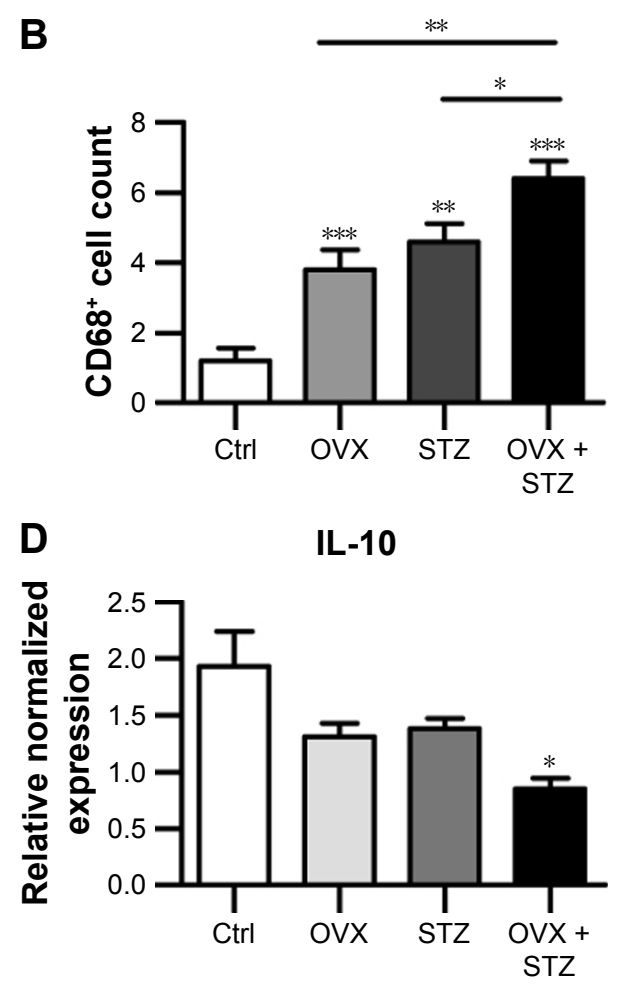
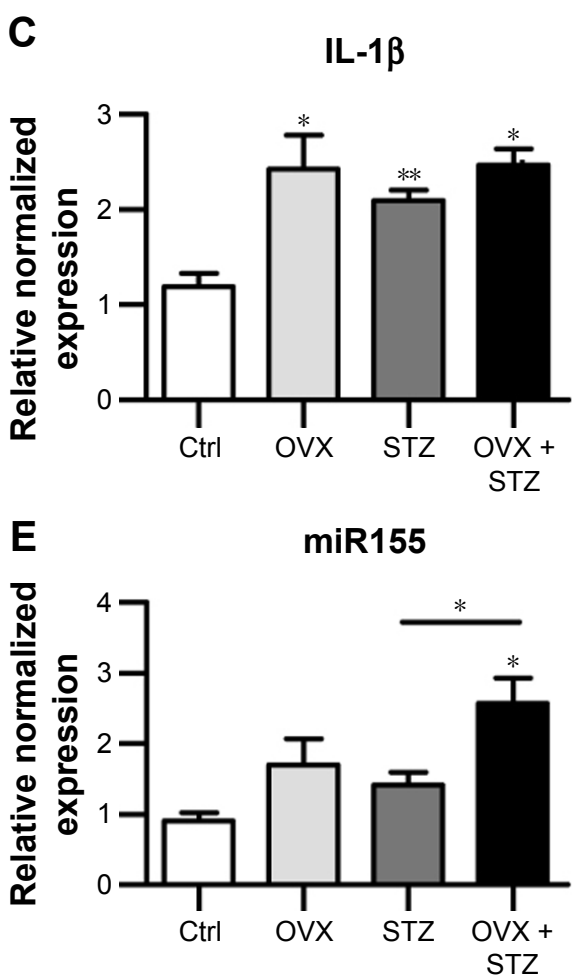

Figure 2 Increased MI polarization in OVX diabetic mice.

Notes: (A) Immunofluorescence confocal images of macrophages (arrowheads) in the hearts from the indicated groups, in which macrophages were stained with antiCD68 (red) and nuclei were stained with Hoechst (blue). Scale bar $=50 \mu \mathrm{m}$ and applies to all images. (B) Quantification of the infiltrated macrophages in the hearts from the indicated groups (mean \pm SEM, analyzed from 4 representative sections from 4 mice in each group). Quantitative PCR data of IL-I $\beta$ (C), IL-I0 (D), and miRI55 (E) expression in the hearts from the indicated groups (mean \pm SEM of 4 mice in each group). $* P<0.05, * * P<0.01$, and $* * * P<0.001$.

Abbreviations: Ctrl, control; Hoechst, Hoechst 33258; IL, interleukin; MI, pro-inflammatory type I macrophage; OVX, ovariectomized; SEM, standard error of the mean; STZ, streptozotocin; PCR, polymerase chain reaction.

We next tested whether macrophage depletion would possibly prevent the aggravation of the cardiac function in the OVX diabetic mice. To this end, macrophages were depleted in the animal models with clophosome (Figure 3A). As expected, injection of clophosome nearly completely and stably depleted the macrophages in the spleen, liver, and heart (Figure 3B-E). However, the cardiac function was not efficiently restored after macrophage depletion, although with a beneficial trend of no statistical significance (Figure 3F and G).

\section{AuNP-mediated miRI55 antagonist delivery promotes $\mathrm{M} 2$ polarization in vivo} Previously, M1 and M2 macrophages were found to play different roles in cardiac homeostasis and repair. ${ }^{23}$ Together with the aforementioned data, we hypothesize that finetuning the balance between M1 and M2, rather than rough depletion of both types, might be beneficial. Previous studies have revealed that miR155 acts as a potent M1 promoter. ${ }^{24}$ Consistent with the previous findings, we also observed increased miR 155 expression in the hearts of OVX diabetic mice (Figure 2E). Accordingly, transfection of miR155 into the murine RAW264.7 macrophages significantly increased IL-1 $\beta$, while reducing the IL-10 expression (Figure 4A-C). In addition, the lysosome staining did not show any significant changes (Figure S3), further confirming the M1-promoting role of miR155. We next explored whether antago-miR155 delivery could be tipping the M1/M2 balance toward M2 in vivo. To deliver miRNA into macrophages, AuNP with a diameter of $10 \mathrm{~nm}$ was included for siRNA conjugation (Figure 4D), as described before. ${ }^{25}$ After conjugation of nucleic acids, the AuNPs were 20-40 nm (Figure 4E), which is the size that could be preferentially phagocytosed by macrophages. To confirm the macrophage-specific delivery, a Cy3-labeled RNA sequence with an 18-nt spacer was conjugated with AuNP, as described in the "Materials and methods" section. Then, $100-\mu \mathrm{L}$ of conjugated AuNP was injected in the tail vein. In vivo AuNP distribution was analyzed 1 hour later. The Cy3 fluorescence signal suggests that the AuNP-mediated miRNA delivery targets the spleen and the liver, where macrophages are abundant, suggesting that the strategy targeting macrophages is highly 


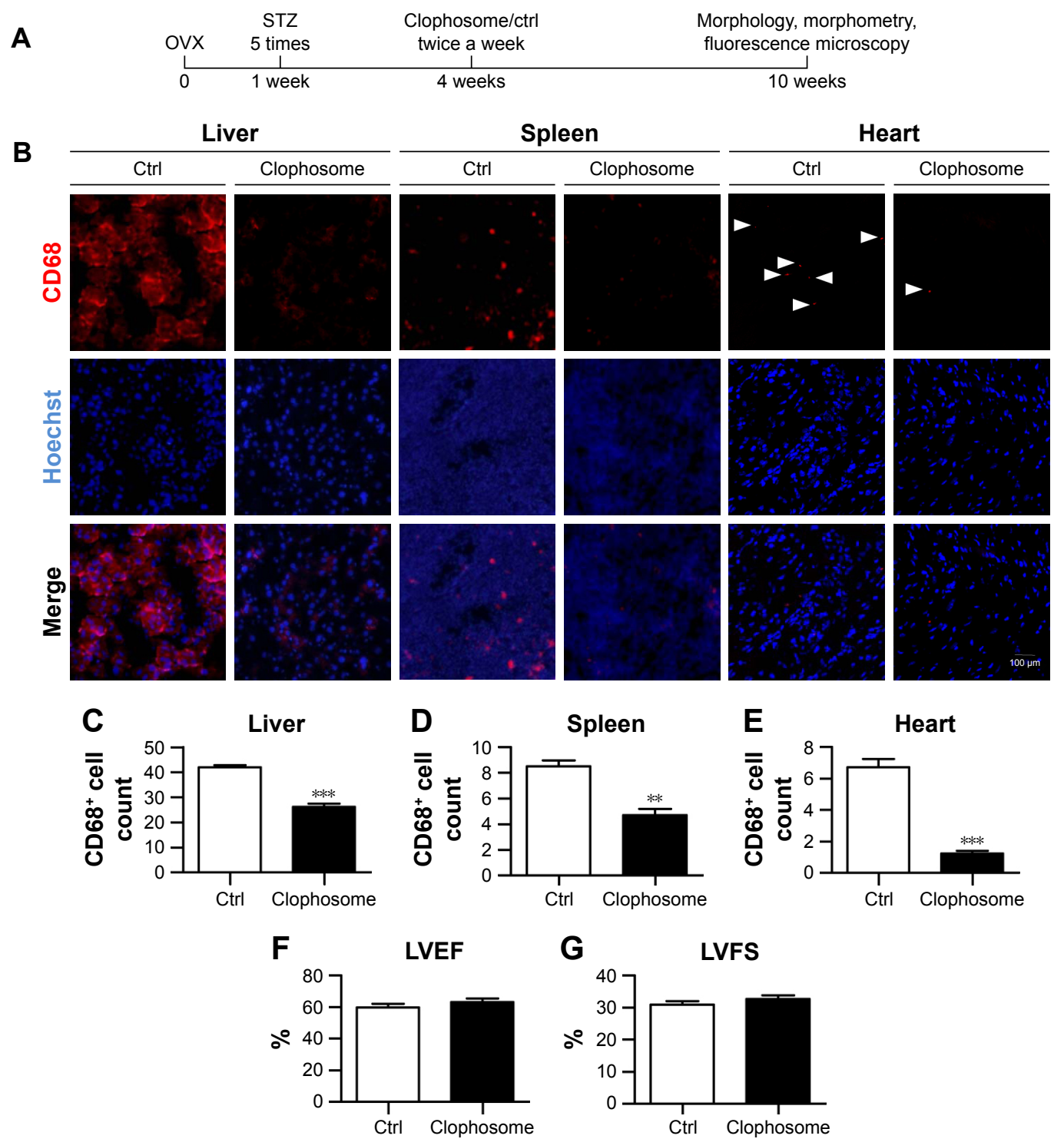

Figure 3 Macrophage depletion marginally rescues the cardiac function in OVX diabetic mice.

Notes: (A) Schematic representation of the experimental procedure. Four weeks after operation, OVX diabetic mice were injected with control or clophosome twice a week for 2 weeks to deplete the macrophages. Ten weeks after operation, cardiac function, morphology, and cellular changes were systematically analyzed. (B) Immunofluorescence confocal images of macrophages in the hearts, livers, and spleens from the indicated groups, in which macrophages were stained with anti-CD68 (red) and nuclei were counterstained with Hoechst (blue). (C-E) Quantitative data of the macrophage percentage in the liver (C), spleen (D), and heart (E) (analyzed from four representative sections from four mice in each group). LVEF (F) and LVFS (G) values of cardiac systolic function in different groups (mean $\pm S D, n=5$ mice in each group). $* * P<0.0$ I and $* * * P<0.00 \mathrm{I}$. Scale bar $=100 \mu \mathrm{m}$ and applies to all images.

Abbreviations: Ctrl, control; Hoechst, Hoechst 33258; LVEF, left ventricular ejection fraction; LVFS, left ventricular fraction shortening; OVX, ovariectomized; SD, standard deviation; STZ, streptozotocin.
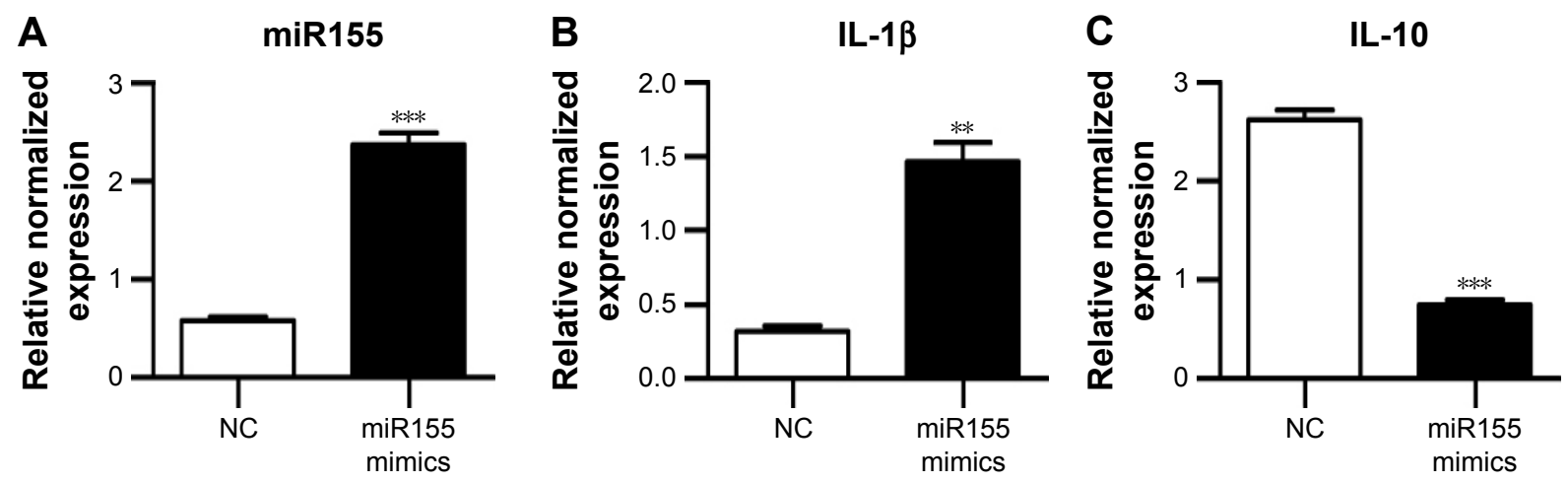

Figure 4 (Continued) 
D

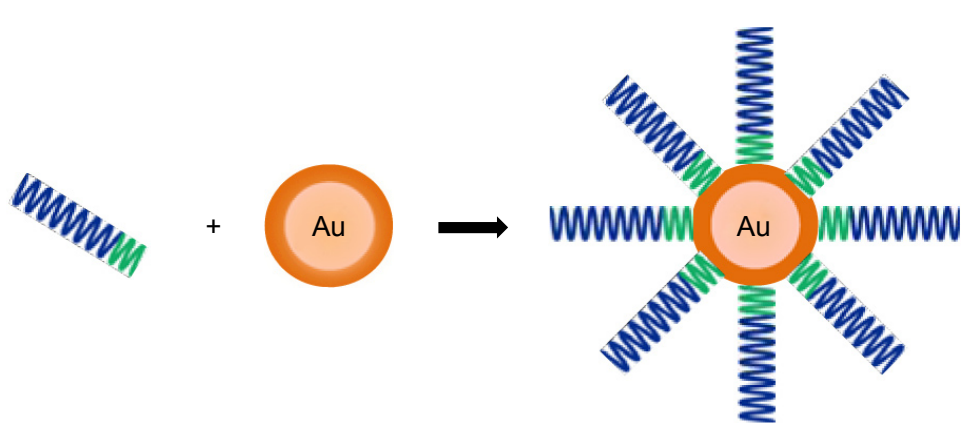

$\mathbf{F}$
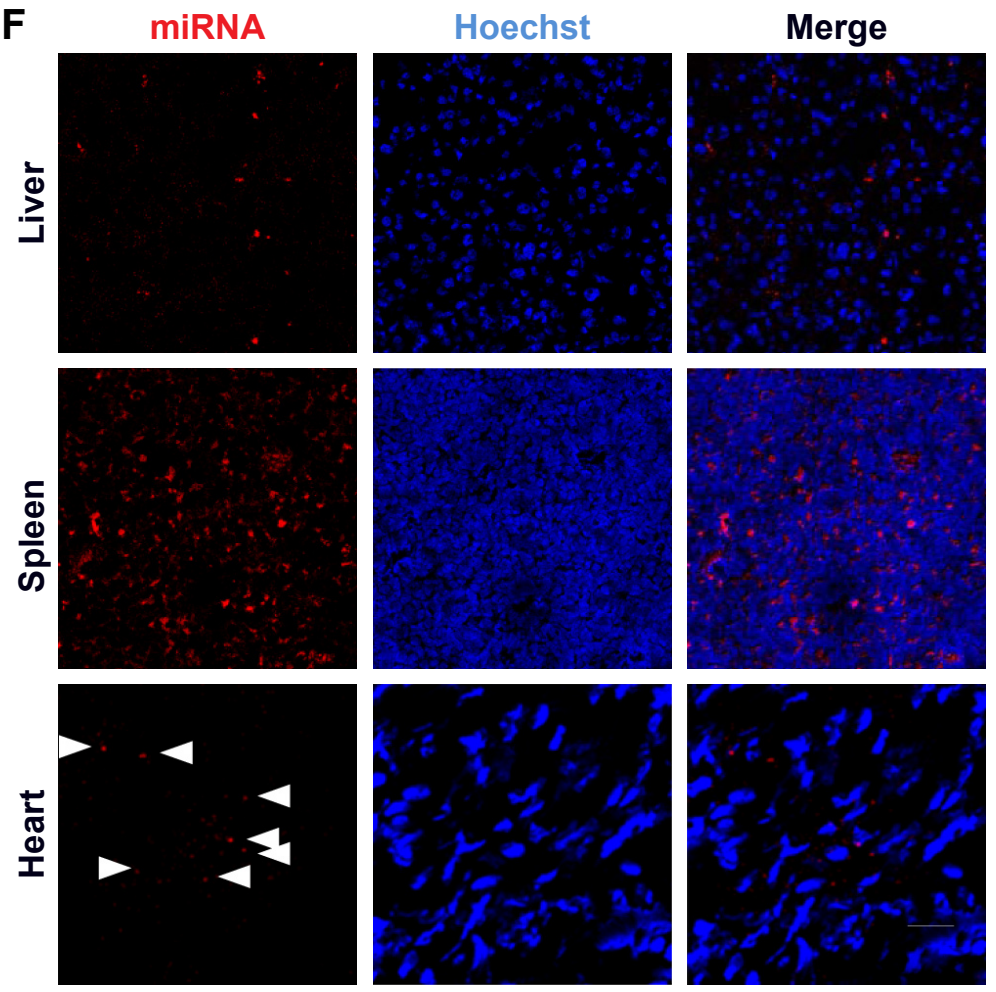

G

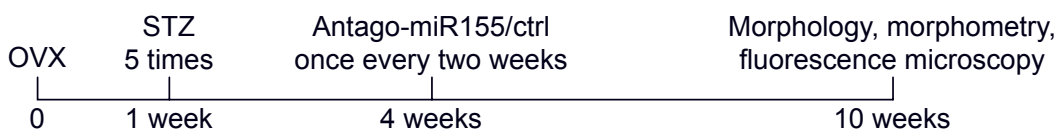

E
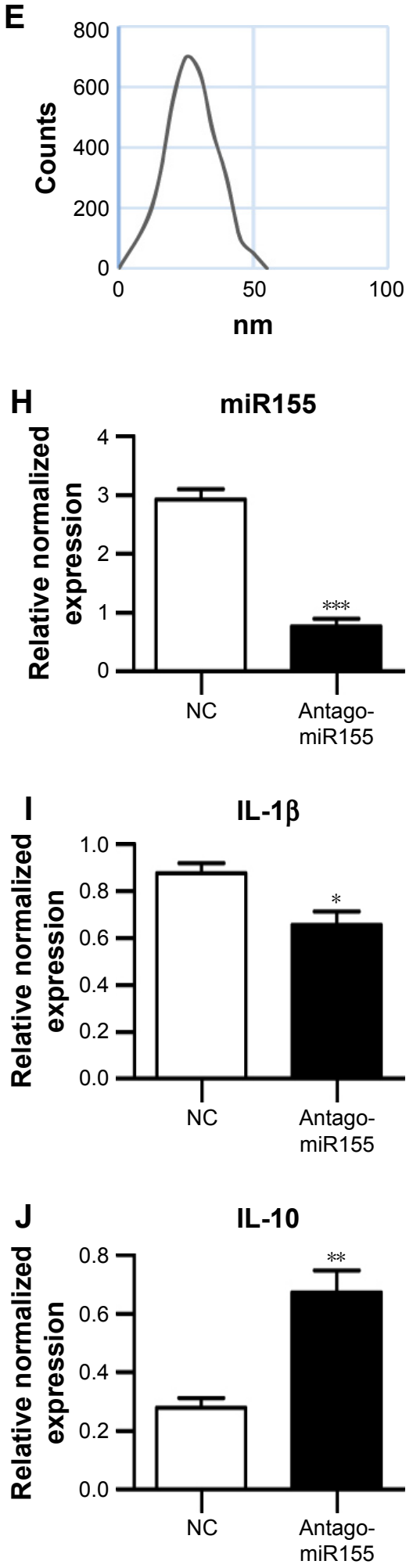

Figure 4 Antago-miR I55-AuNP targets macrophages with high specificity in vivo.

Notes: (A-C) RAW264.7 cells were transfected with miRI55 mimics by lipofectamine 2000. Expression of miRI55 (A), IL-I $\beta$ (B), and IL-I0 (C) was analyzed by qPCR (mean \pm SEM of three experiments). (D) Synthesis of miRNA-conjugated AuNP nanoparticles. Thiolated miRNA antagonist was added to solutions of citrate-stabilized gold colloid and attached through thiol-gold chemistry. (E) Size distribution of the conjugated AuNP analyzed by NTA. (F) Immunofluorescence confocal images of miRNA-AuNP in the livers, spleens, and hearts from the indicated groups, in which miRNA-AuNP was labeled with Cy3 (red) and nuclei were counterstained with Hoechst (blue). Scale bar $=50 \mu \mathrm{m}$ and applies to all images. (G) Schematic representation of the experimental procedure. OVX diabetic mice 4 weeks after operation were injected with control or antago-miRI55 AuNP once a week for 2 weeks. Ten weeks after operation, cardiac function, morphology, and cellular changes were systematically analyzed. (H-J) qPCR data of miRI55 (H), IL-I $\beta(\mathbf{I})$, and IL-I0 (J) expression in the hearts of OVX diabetic mice with NC or miRI55 antagonist delivery (mean \pm SEM of 3 mice in each group). $* P<0.05, * * P<0.01$, and $* * * P<0.001$.

Abbreviations: AuNP, gold nanoparticles; Hoechst, Hoechst 33258; IL-I $\beta$, interleukin-I $\beta$; IL-I0, interleukin- I0; NC, negative control; NTA, nanoparticle tracking analysis; OVX, ovariectomized; qPCR, quantitative polymerase chain reaction; SEM, standard error of the mean; STZ, streptozotocin. 
specific (Figure 4F). Notably, there were also sparse Cy3 signals in the heart, suggesting that AuNP could be delivered into both resident and exogenous macrophages of the heart (Figure 4F).

Next, both negative control and antago-miR155 were conjugated with AuNP and delivered into the OVX diabetic mouse, followed by indicated analysis (Figure 4G). After in vivo antago-miR155 delivery, the macrophage number in the heart did not change significantly (Figure S4A). However, antago-miR155 delivery reduced the M1 marker IL-1 $\beta$ expression, while increasing the M2 marker IL-10 in the heart (Figure $4 \mathrm{H}-\mathrm{J}$ ), consistent with the in vitro data.

\section{AuNP-mediated antago-miR I 55 delivery restored the cardiac function}

With the M2 polarization and decreased inflammation, cardiac hypertrophy and fibrosis were also partially reversed by miR155-AuNP, as indicated by WGA staining and Masson
Trichrome staining (Figure 5A-D; Figure S4B). Accordingly, miR155-AuNP reduced the cellular apoptosis (Figure 5E, F). Besides, miR155-AuNP also increased the vascular density in the OVX diabetic group (Figure S4C). Consistent with the molecular changes, miR155-AuNP injection in the OVX diabetic mice significantly improved the cardiac function, as shown by increased LVEF and LVFS (Figure 5G, H). Taken together, all of the above study findings revealed that a central role of miR155-M1/M2 imbalance in cardiomyopathy in the OVX diabetic mouse model, and AuNP-based antago-miR155 delivery, specifically and efficiently promoted $\mathrm{M} 2$ polarization, which in turn restored the cardiac function by coordinating inflammation, apoptosis, and fibrosis (Figure 6).

\section{Discussion}

By using the OVX diabetic mouse model, we revealed for the first time that upregulation of miR155 results in excessive polarized M1 macrophages and thus aggravates inflammation
A
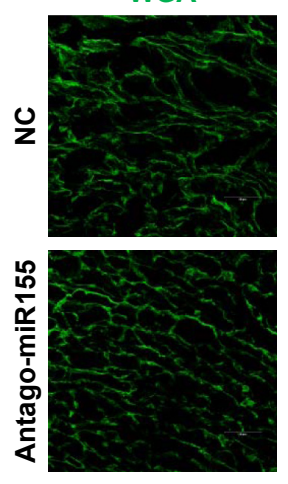

E
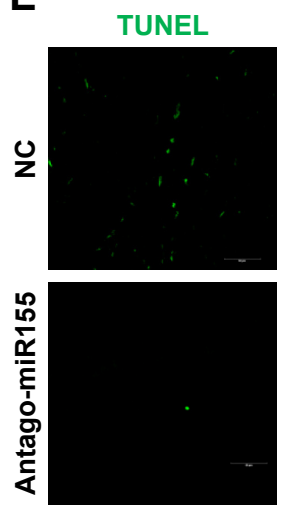

Hoechst
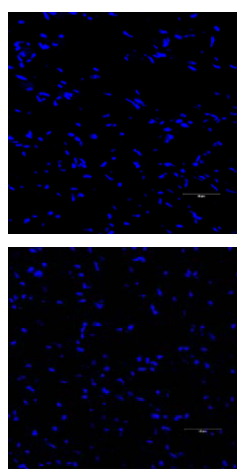

Hoechst
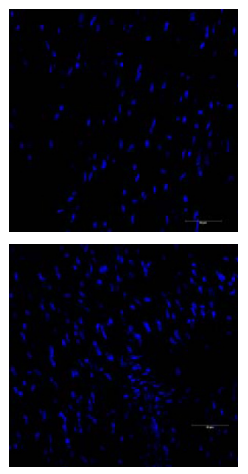

Merge
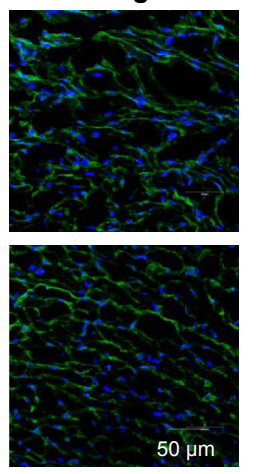

B
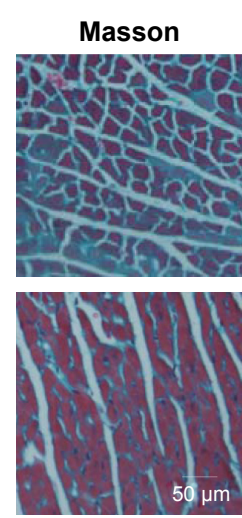

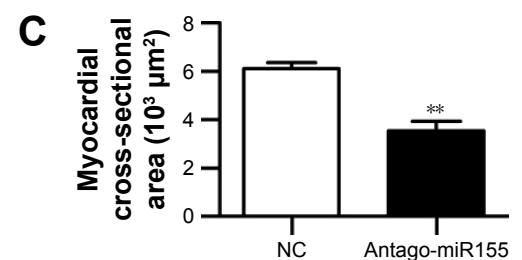

D

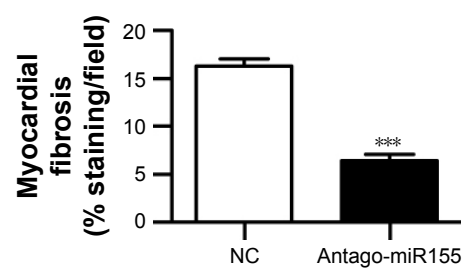

Figure 5 Antago-miRI55-AuNP rescues the cardiac function in ovariectomized diabetic mice.

Notes: (A) Representative WGA-stained sections from the left ventricular mid-chamber of the above mice. (B) Representative images of the Masson staining of ctrl and antago-miR 155 AuNP-treated heart. (C) Quantitative data of the cardiomyocyte size in the indicated groups ( $n=4)$. (D) Quantitative data of the fibrotic area in the indicated groups (mean \pm SEM, $n=4$ mice in each group). (E) Apoptotic cells in the hearts of ovariectomized diabetic mice after control and antago-miR 155 treatment were probed by TUNEL staining. (F) Quantitative data of the apoptotic cells in the indicated groups $(n=4)$. LVEF $(\mathbf{G})$ and LVFS $(\mathbf{H})$ values of cardiac systolic function in the aforementioned mice (mean $\pm S D, n=5$ mice in each group). Scale bars $=50 \mu \mathrm{m}$ and apply to all images. $* * P<0.01$, $* * * P<0.001$.

Abbreviations: AuNP, gold nanoparticle; Ctrl, control; Hoechst, Hoechst 33258; LVEF, left ventricular ejection fraction; LVFS, left ventricular fraction shortening; NC, negative control; SD, standard deviation; TUNEL, terminal deoxynucleotidyl transferase mediated dUTP nick end labeling; WGA, wheat germ agglutinin. 

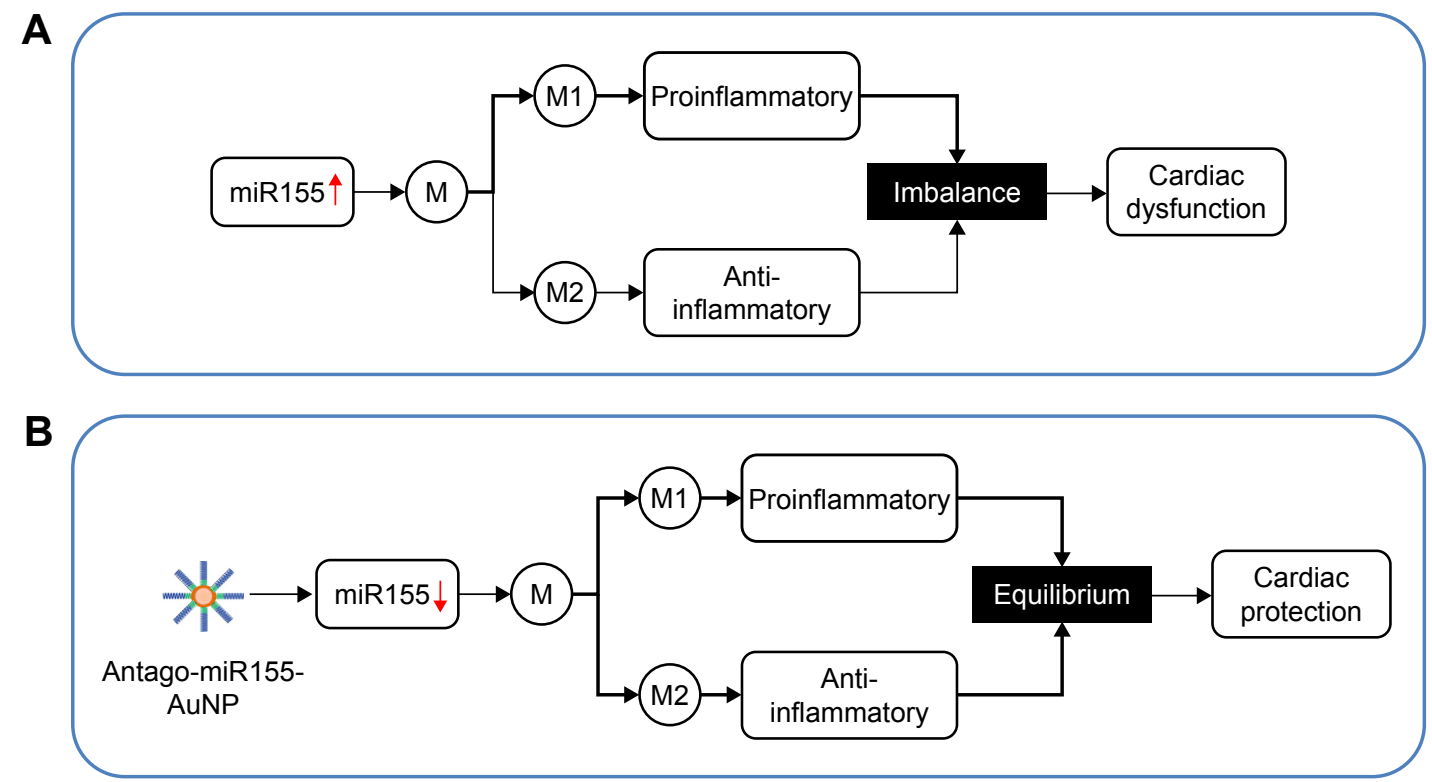

Figure 6 Graphical summary of the study.

Note: (A) Excessive miRI55 in the OVX diabetic mouse model causes MI/M2 imbalance, which plays a central role in cardiomyopathy in the OVX diabetic mouse model. (B) AuNP-based miR 155 inhibitor (antago-miRI55) delivery efficiently promotes M2 polarization, which in turn restores the cardiac function by coordinating inflammation, apoptosis, angiogenesis, and fibrosis.

Abbreviations: AuNP, gold nanoparticle; M, macrophages; MI, proinflammatory type I macrophages; M2, anti-inflammatory type 2 macrophages; OVX, ovariectomized.

in the heart, which leads to exaggerated cardiomyopathy. The above mechanism might explain the increased cardiovascular disease incidence and poor prognosis for postmenopausal diabetic patients. Moreover, we have shown that AuNP mediated cell specific inhibition of miR155 expression in macrophages promotes M2 polarization, represses inflammation, and thus restores the cardiac function.

Both the resident cardiac macrophages and exogenous macrophages play an important role in the cardiac homeostasis upon cardiac injury. ${ }^{26}$ First of all, macrophages are essential to clear the cellular debris due to cardiac injury. ${ }^{27}$ Second, macrophages, both resident and exogenous, express a number of proangiogenic factors, such as Rentla/Fizz1, IGF-1, and VEGF, to promote angiogenesis and thus limit cell death because of a lack of blood supply. ${ }^{26,28}$ Third, macrophages, mainly M2, inhibit the inflammation, which in turn limits the damage induced by the reactive oxygen species. Last, but not least, both resident and exogenous macrophages secrete a number of profibrotic proteins to induce fibroblast proliferation and collagen deposition by both TGF $\beta 1$-dependent and independent pathways. Particularly, M2 macrophages have a potent profibrogenic function. ${ }^{23}$ Notably, debris clearance, angiogenesis, inflammation, and fibrosis play different, even opposite, roles in the cardiac damage repair. All of these data suggest that macrophage contribution, particularly M2 contribution, to the cardiac homeostasis is likely to change with age, hormone status, damage duration, and intensity.
In other words, tuning or modulating macrophages toward better prognosis should be context dependent. Our current study revealed for the first time that in the context of OVX diabetic mice, excessive M1 over M2 should be responsible for the aggravated damage to cardiac function, which is at least partially consistent with the notion that excessive inflammation accounts for diabetic cardiomyopathy. ${ }^{29-31}$ In short, besides inflammation and angiogenesis regulation, macrophages are considered as the housekeeping cells involved in the detection of foreign antigens and danger signatures, and the clearance of tissue debris. Refinement of the balance of M1/M2 would promote damage repair toward the better prognosis. Our study proposes a good model that therapeutic delivery miRNA mimics or inhibitors would be a promising strategy for tuning macrophage polarization and thus management of related diseases.

Usually, the nanoparticle-based gene/drug delivery strategies target cells like epithelium or cardiomyocytes, and thus phagocytosis by macrophages should be avoided. In contrast, the therapeutic targeting of macrophages is relatively easy and specific. ${ }^{32,33}$ AuNP is an ideal material for nucleic acid delivery. ${ }^{14,15}$ The AuNPs that are coated with nucleotide acids are recruited to the scavenger receptors on the cells, especially the macrophages, facilitating phagocytosis, which is responsible for efficient and superior delivery. ${ }^{34}$ AuNP can be fabricated in a scalable fashion, and the cytotoxicity, ${ }^{19,20}$ biodistribution, ${ }^{21}$ and in vivo excretion properties can be 
fine-tuned by size control. ${ }^{22}$ To this end, an optimized AuNP size is still needed to improve safety and efficacy. Currently, the functionalized AuNP surface allows small nucleic acids to be conjugated on the particle surface, ${ }^{17,18}$ making siRNAs or miRNA inhibitors rational therapeutic drugs for AuNP strategy. Although AuNP has high biocompatibility, the fact of how the AuNP nanoparticles exit cells and are cleared from the body is currently largely unknown. It is believed that the AuNPs are cleared from the kidney and in vivo retention is considered as the main cause of the side effects. ${ }^{35}$ Notably, besides AuNP, multiple nanoparticles are made up of other available materials. For example, both poly (lactic-co-glycolic acid) (PLGA) nanoparticles and chitosan nanoparticles are the most successfully developed polymeric nanoparticles, due to their attractive biodegradability and biocompatibility. ${ }^{36-38}$ It is thus interesting to test whether PLGA- or chitosan-based nanoparticles would produce similar and even better beneficial results.

In summary, we revealed that excessive inflammation, at least partially due to overactivated M1 macrophages, accounts for the exaggerated cardiac defects. Moreover, AuNP covalently conjugated with thiol-modified antagomiR155, preferentially delivered the nucleic acids to the macrophages, and restored the cardiac function. Our study suggests that therapeutically reducing miR155 by AuNP in macrophages would serve as a promising strategy in improving the cardiac function in postmenopausal diabetic patients and/or others with similar etiology.

\section{Acknowledgments}

This study was funded by the Social Development Guide Plan of Science and Technology Department of Xi' an (SF1511(7) to Jing Ma) and the National Science Foundation of China (NSFC81271579 to Hui Chen). We greatly appreciate the technical help with gold nanoparticle manipulation from Xiangwei Liu.

\section{Disclosure}

The authors report no conflicts of interest in this work.

\section{References}

1. Schierbeck L. Primary prevention of cardiovascular disease with hormone replacement therapy. Climacteric. 2015;18(4):492-497.

2. Staszewsky L, Cortesi L, Baviera M, et al. Diabetes mellitus as risk factor for atrial fibrillation hospitalization: Incidence and outcomes over nine years in a region of Northern Italy. Diabetes Res Clin Pract. 2015;109(3): 476-484.

3. Schmiegelow MD, Hedlin H, Stefanick ML, et al. Insulin resistance and risk of cardiovascular disease in postmenopausal women a cohort study from the women's health initiative. Circ Cardiovasc Qual Outcomes. 2015;8(3):309-316.
4. Casey BA, Kohrt WM, Schwartz RS, Van Pelt RE. Subcutaneous adipose tissue insulin resistance is associated with visceral adiposity in postmenopausal women. Obesity (Silver Spring). 2014;22(6): $1458-1463$.

5. Fernandez-Velasco M, Gonzalez-Ramos S, Bosca L. Involvement of monocytes/macrophages as key factors in the development and progression of cardiovascular diseases. Biochem J. 2014;458(2):187-193.

6. Zheng C, Yang Q, Cao J, et al. Local proliferation initiates macrophage accumulation in adipose tissue during obesity. Cell Death Dis. 2016; $7: \mathrm{e} 2167$.

7. O'Shea JJ, Paul WE. Mechanisms underlying lineage commitment and plasticity of helper CD4+T cells. Science. 2010;327(5969): 1098-1102.

8. Mills CD. M1 and M2 macrophages: oracles of health and disease. Crit Rev Immunol. 2012;32(6):463-488.

9. Mills CD, Ley K. M1 and M2 macrophages: the chicken and the egg of immunity. J Innate Immun. 2014;6(6):716-726.

10. Roma-Lavisse C, Tagzirt M, Zawadzki C, et al. M1 and M2 macrophage proteolytic and angiogenic profile analysis in atherosclerotic patients reveals a distinctive profile in type 2 diabetes. Diab Vasc Dis Res. 2015; 12(4):279-289.

11. Bartel DP. MicroRNAs: target recognition and regulatory functions. Cell. 2009;136(2):215-233.

12. Martinez-Nunez RT, Louafi F, Sanchez-Elsner T. The interleukin 13 (IL-13) pathway in human macrophages is modulated by microRNA155 via direct targeting of interleukin 13 receptor alpha1 (IL13Ralpha1). J Biol Chem. 2011;286(3):1786-1794.

13. Corsten MF, Papageorgiou A, Verhesen W, et al. MicroRNA profiling identifies microRNA-155 as an adverse mediator of cardiac injury and dysfunction during acute viral myocarditis. Circ Res. 2012;111(4): 415-155.

14. Boisselier E, Astruc D. Gold nanoparticles in nanomedicine: preparations, imaging, diagnostics, therapies and toxicity. Chem Soc Rev. 2009; 38(6):1759-1782.

15. Gindy ME, Prud'homme RK. Multifunctional nanoparticles for imaging, delivery and targeting in cancer therapy. Expert Opin Drug Deliv. 2009;6(8):865-878

16. Daniel MC, Astruc D. Gold nanoparticles: assembly, supramolecular chemistry, quantum-size-related properties, and applications toward biology, catalysis, and nanotechnology. Chem Rev. 2004;104(1): 293-346.

17. Bowman MC, Ballard TE, Ackerson CJ, Feldheim DL, Margolis DM, Melander C. Inhibition of HIV fusion with multivalent gold nanoparticles. J Am Chem Soc. 2008;130(22):6896-6897.

18. Ryan JA, Overton KW, Speight ME, et al. Cellular uptake of gold nanoparticles passivated with BSA-SV40 large T antigen conjugates. Anal Chem. 2007;79(23):9150-9159.

19. Kim ST, Saha K, Kim C, Rotello VM. The role of surface functionality in determining nanoparticle cytotoxicity. Acc Chem Res. 2013;46(3): 681-691.

20. Chompoosor A, Saha K, Ghosh PS, et al. The role of surface functionality on acute cytotoxicity, ROS generation and DNA damage by cationic gold nanoparticles. Small. 2010;6(20):2246-2249.

21. De Jong WH, Hagens WI, Krystek P, Burger MC, Sips AJ, Geertsma RE. Particle size-dependent organ distribution of gold nanoparticles after intravenous administration. Biomaterials. 2008;29(12): 1912-1919.

22. Zhu M, Perrett S, Nie G. Understanding the particokinetics of engineered nanomaterials for safe and effective therapeutic applications. Small. 2013;9(9-10):1619-1634.

23. Fujiu K, Wang J, Nagai R. Cardioprotective function of cardiac macrophages. Cardiovasc Res. 2014;102(2):232-239.

24. Cai X, Yin Y, Li NZ, et al. Re-polarization of tumor-associated macrophages to pro-inflammatory M1 macrophages by microRNA-155. J Mol Cell Biol. 2012;4(5):341-343.

25. Zheng D, Giljohann DA, Chen DL, et al. Topical delivery of siRNAbased spherical nucleic acid nanoparticle conjugates for gene regulation. Proc Natl Acad Sci U S A. 2012;109(30):11975-11980. 
26. Pinto AR, Godwin JW, Rosenthal NA. Macrophages in cardiac homeostasis, injury responses and progenitor cell mobilisation. Stem Cell Res. 2014;13(3 Pt B):705-714.

27. Pinto AR, Godwin JW, Chandran A, et al. Age-related changes in tissue macrophages precede cardiac functional impairment. Aging (Albany NY). 2014;6(5):399-413.

28. Nucera S, Biziato D, De Palma M. The interplay between macrophages and angiogenesis in development, tissue injury and regeneration. Int J Dev Biol. 2011;55(4-5):495-503.

29. Mauvais-Jarvis F, Clegg DJ, Hevener AL. The role of estrogens in control of energy balance and glucose homeostasis. Endocr Rev. 2013;34(3): 309-338

30. Shapiro Y, Mashavi M, Luckish E, Shargorodsky M. Diabetes and menopause aggravate age-dependent deterioration in arterial stiffness. Menopause. 2014;21(11):1234-1238.

31. Mascarenhas-Melo F, Marado D, Palavra F, et al. Diabetes abrogates sex differences and aggravates cardiometabolic risk in postmenopausal women. Cardiovasc Diabetol. 2013;12:61.

32. Weissleder R, Nahrendorf M, Pittet MJ. Imaging macrophages with nanoparticles. Nat Mater. 2014;13(2):125-138.

33. Lameijer MA, Tang J, Nahrendorf M, Beelen RHJ, Mulder WJM. Monocytes and macrophages as nanomedicinal targets for improved diagnosis and treatment of disease. Expert Rev Mol Diagn. 2013; 13(6):567-580.

34. Rosi NL, Giljohann DA, Thaxton CS, Lytton-Jean AK, Han MS, Mirkin CA. Oligonucleotide-modified gold nanoparticles for intracellular gene regulation. Science. 2006;312(5776):1027-1030.

35. Khlebtsov N, Dykman L. Biodistribution and toxicity of engineered gold nanoparticles: a review of in vitro and in vivo studies. Chem Soc Rev. 2011;40(3):1647-1671.
36. Martin-Banderas L, Duran-Lobato M, Munoz-Rubio I, Alvarez-Fuentes J, Fernandez-Arevalo M, Holgado MA. Functional PLGA NPs for oral drug delivery: recent strategies and developments. Mini Rev Med Chem. 2013;13(1):58-69.

37. Lai P, Daear W, Lobenberg R, Prenner EJ. Overview of the preparation of organic polymeric nanoparticles for drug delivery based on gelatine, chitosan, poly(day,l-lactide-co-glycolic acid) and polyalkylcyanoacrylate. Colloids Surf B Biointerfaces. 2014;118:154-163.

38. Kumari A, Yadav SK, Yadav SC. Biodegradable polymeric nanoparticles based drug delivery systems. Colloids Surf B Biointerfaces. 2010;75(1):1-18.

39. Lizotte E, Grandy SA, Tremblay A, Allen BG, Fiset C. Expression, distribution and regulation of sex steroid hormone receptors in mouse heart. Cell Physiol Biochem. 2009;23(1-3):75-86.

40. Hunt DL, Campbell PH, Zambon AC, et al. Early postmyocardial infarction survival in Murphy Roths Large mice is mediated by attenuated apoptosis and inflammation but depends on genetic background. Exp Physiol. 2012;97(1):102-114.

41. Grabner A, Amaral AP, Schramm K, et al. Activation of cardiac fibroblast growth factor receptor 4 causes left ventricular hypertrophy. Cell Metab. 2015;22(6):1020-1032.

42. Fan D, Takawale A, Shen M, et al. Cardiomyocyte a disintegrin and metalloproteinase 17 (ADAM17) is essential in post-myocardial infarction repair by regulating angiogenesis. Circ Heart Fail. 2015;8(5): 970-979.

43. Anders $\mathrm{S}$, Huber W. Differential expression analysis for sequence count data. Genome Biol. 2010;11(10):R106.

44. Pryor PR. Imaging and spectroscopic analysis of living cells. In: Michael Conn P, editor. Methods In Enzymology. Amsterdam: Elsevier Inc; 2012;505:145-157. 


\section{Supplementary materials}

A
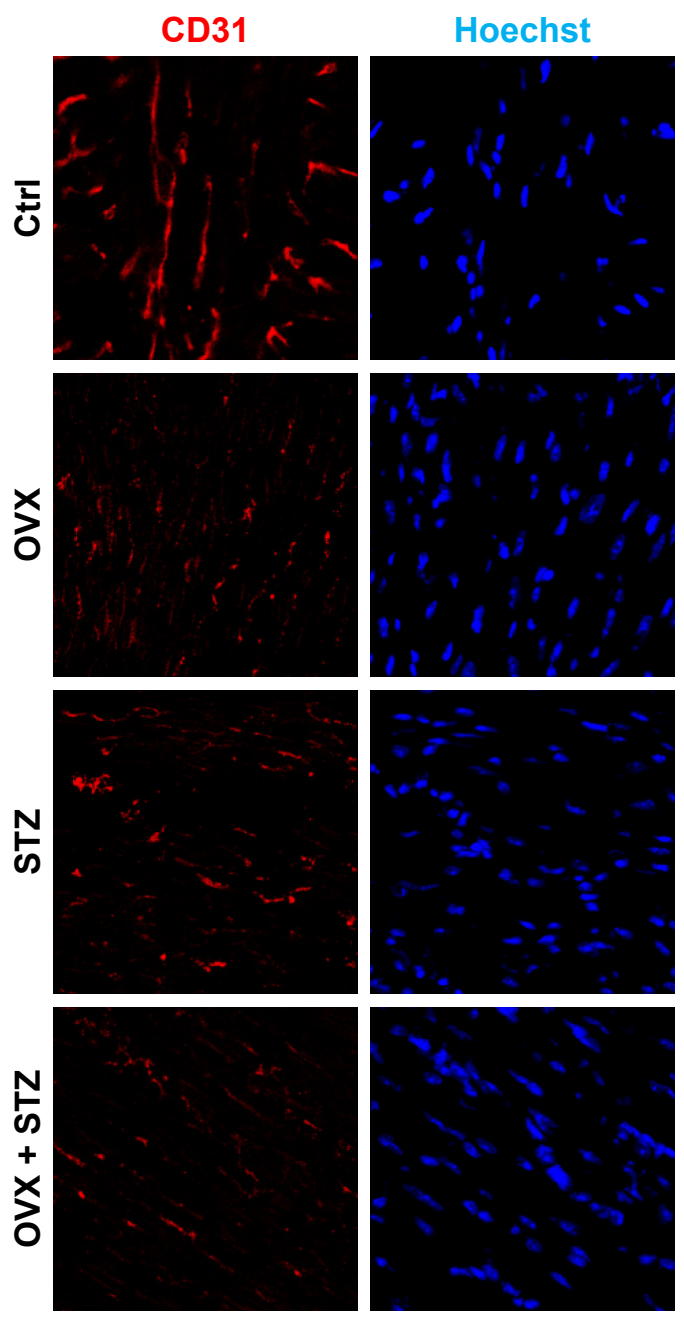

B

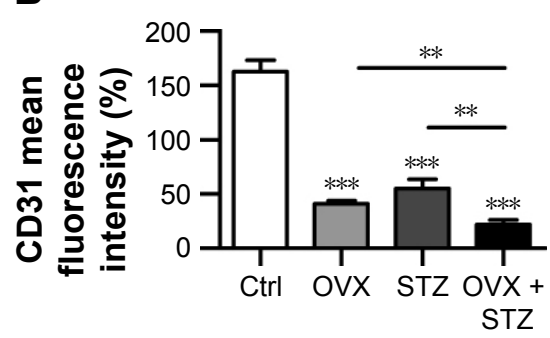

Merge
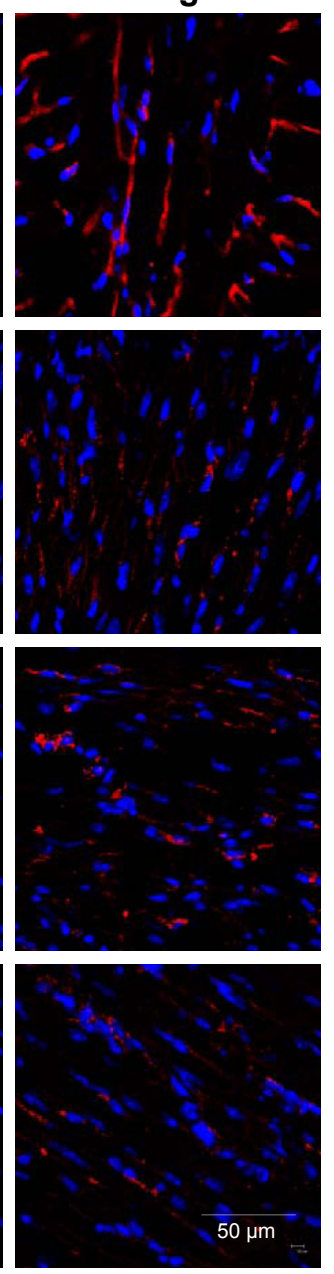

$0 \mu \mathrm{m}$

Figure SI Decreased blood vessel intensity in the hearts of OVX diabetic mice.

Notes: (A) Endothelial cells were stained with anti-CD3I (red) and nuclei were counterstained with Hoechst (blue). Scale bar $=50 \mu \mathrm{m}$ and applies to all images. (B) Quantification of the blood vessel intensity in the above groups (mean $\pm \mathrm{SEM}$, data were analyzed from four sections from four mice in each group, $* * P<0.0 \mathrm{I}$ and $* * * P<0.00 \mathrm{I}$ ). Abbreviations: Ctrl, control; Hoechst, Hoechst 33258; OVX, ovariectomized; SEM, standard error of the mean; STZ, streptozotocin. 

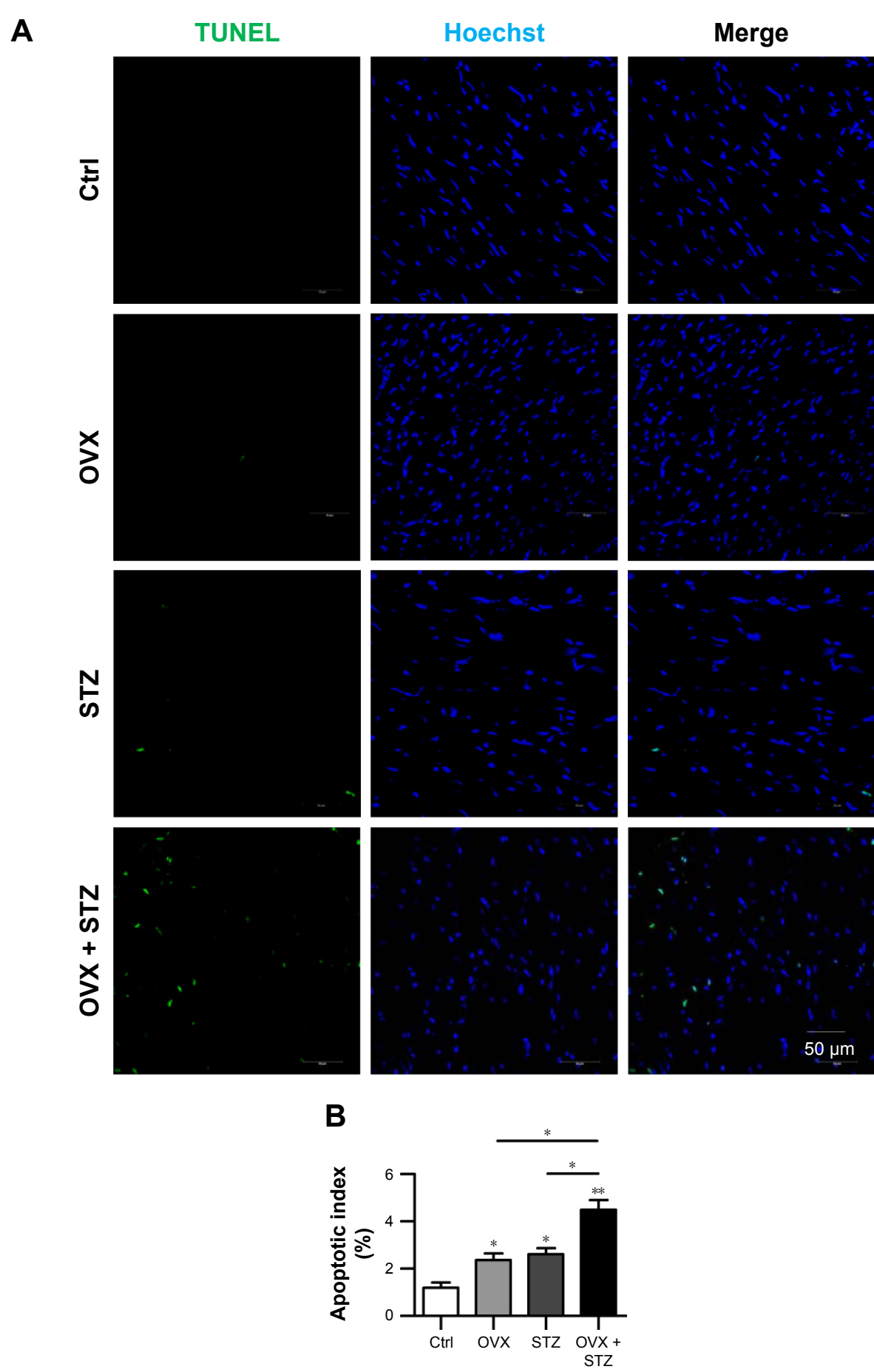

Figure S2 Ovariectomy further increases cell apoptosis in the hearts of diabetic mice.

Notes: (A) Apoptotic cells in the hearts of indicated groups of mice were probed by TUNEL staining. (B) Quantitative data of the apoptotic cells in the indicated groups (mean \pm SEM, data were analyzed from four sections from four mice in each group, $* P<0.05$ and $* * P<0.01$ ).

Abbreviations: Ctrl, control; Hoechst, Hoechst 33258; OVX, ovariectomized; SEM, standard error of the mean; STZ, streptozotocin; TUNEL, terminal deoxynucleotidyl transferase mediated dUTP nick end labeling. 

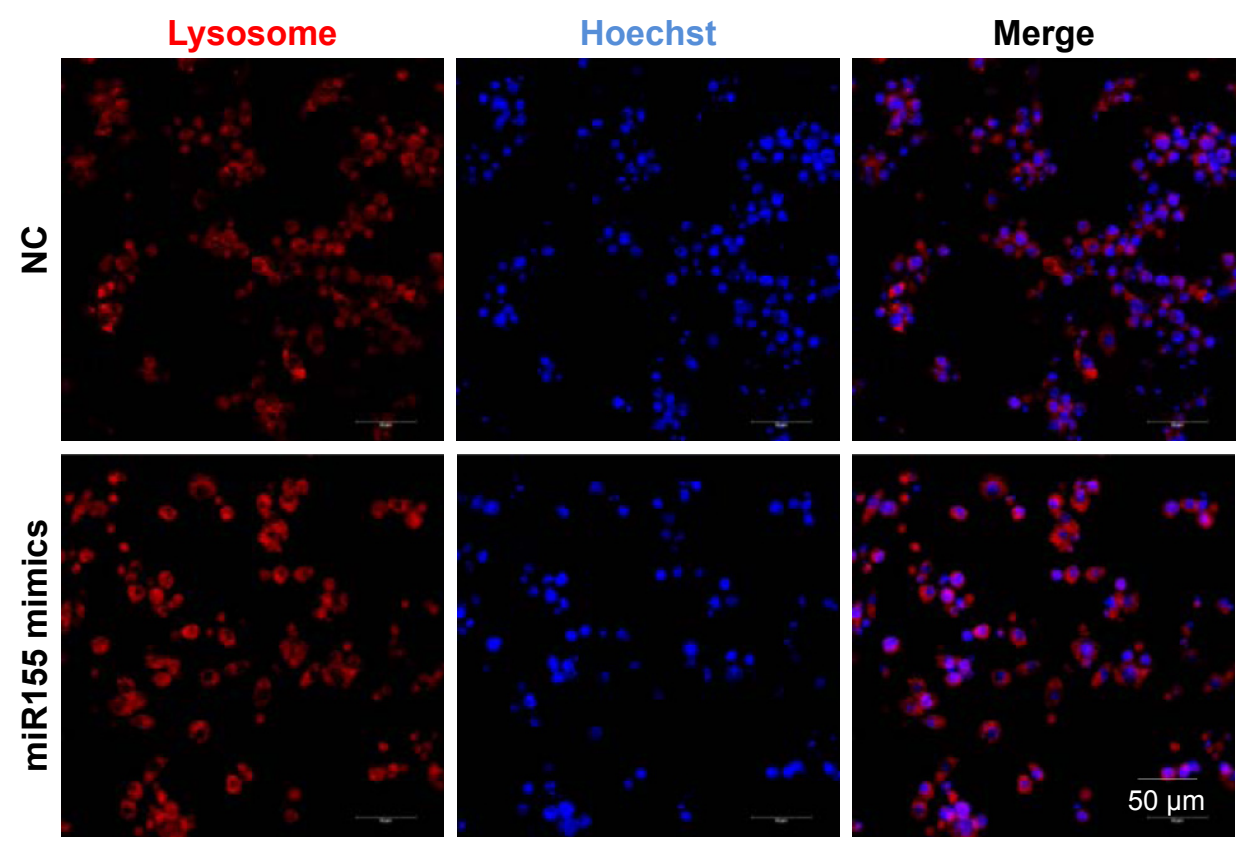

Figure S3 miRI55-AuNP has minimal effects on lysosome.

Notes: RAW264.7 cells were transfected with NC and miR I 55 mimics, respectively. Forty-eight hours later, cells were labeled with Lyso-Tracker Red (red) and counterstained with Hoechst (blue). Scale bar $=50 \mu \mathrm{m}$ and applies to all images.

Abbreviations: AuNP, gold nanoparticle; Hoechst, Hoechst 33258; NC, negative control.

A
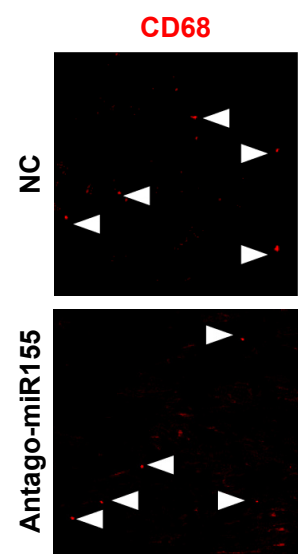

Hoechst
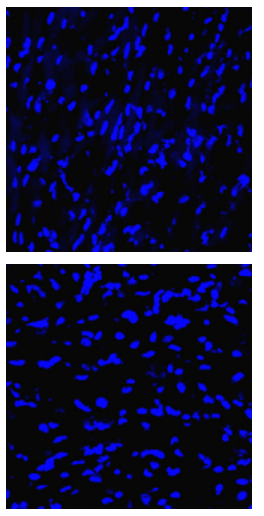

Masson

B
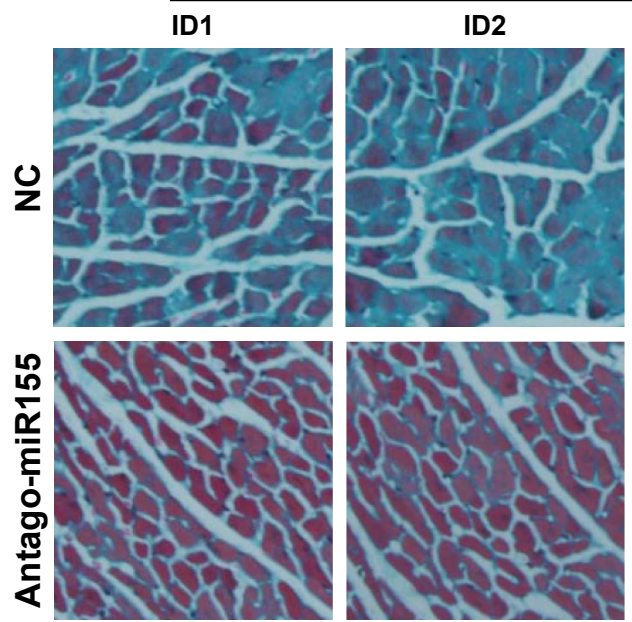

ID3
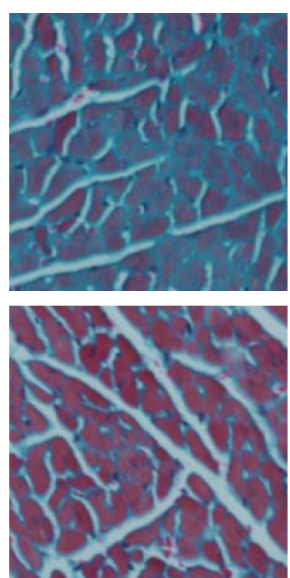

Merge
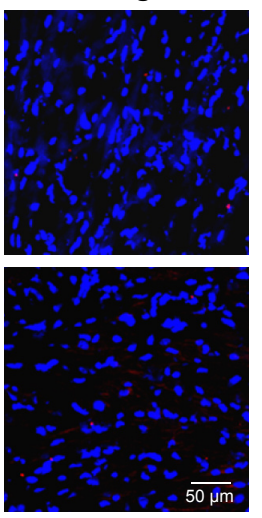

Figure S4 (Continued) 
C
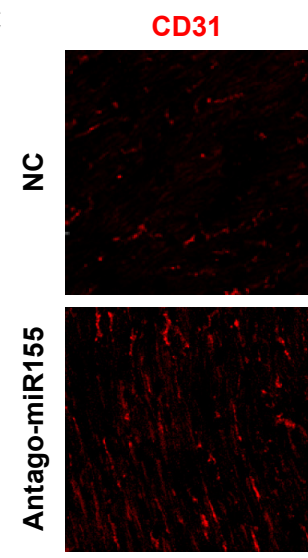

Hoechst
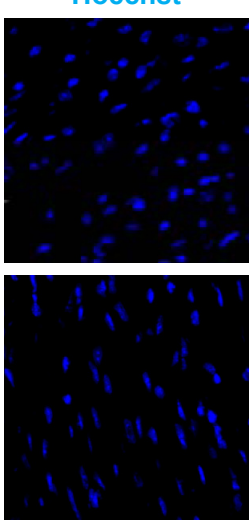

Merge
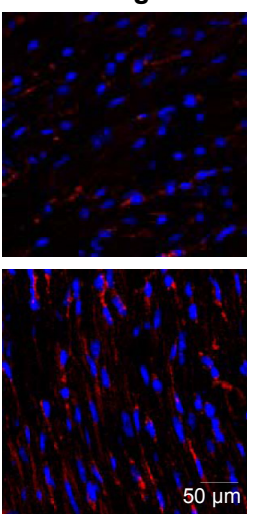

Figure S4 Antago-miR I55-AuNP increases endothelial intensity and rescues the cardiac function in ovariectomized diabetic mice.

Notes: (A) Immunofluorescence confocal images of macrophages in the hearts from the NC and antago-miRI55 groups, in which macrophages were stained with antiCD68 (red) and nuclei, were counterstained with Hoechst (blue). Scale bar $=50 \mu \mathrm{m}$ and applies to all images. (B) Representative images of the Masson Trichrome staining of the hearts from the five mice of each group. Scale bar $=50 \mu \mathrm{m}$ and applies to all images. (C) Immunofluorescence confocal images of endothelial cells in the hearts from the NC and antago-miR I55 groups. Endothelial cells were stained with anti-CD3I (red) and nuclei were counterstained with Hoechst (blue). Scale bar $=50 \mu \mathrm{m}$ and applies to all images.

Abbreviations: AuNP, gold nanoparticle; Hoechst, Hoechst 33258; NC, negative control.

\section{Publish your work in this journal}

The International Journal of Nanomedicine is an international, peerreviewed journal focusing on the application of nanotechnology in diagnostics, therapeutics, and drug delivery systems throughout the biomedical field. This journal is indexed on PubMed Central, MedLine, CAS, SciSearch $\AA$, Current Contents ${ }^{\circledR} /$ Clinical Medicine,
Journal Citation Reports/Science Edition, EMBase, Scopus and the Elsevier Bibliographic databases. The manuscript management system is completely online and includes a very quick and fair peer-review system, which is all easy to use. Visit http://www.dovepress.com/ testimonials.php to read real quotes from published authors.

\footnotetext{
Submit your manuscript here: http://www.dovepress.com/international-journal-of-nanomedicine-journal
} 